\title{
Strong-Stability-Preserving, K-Step, 5- to 10-Stage, Hermite-Birkhoff Time-Discretizations of Order 12
}

\author{
Truong Nguyen-Ba, Huong Nguyen-Thu, Re'mi Vaillancourt \\ Department of Mathematics and Statistics, University of Ottawa, Ottawa, Canada \\ E-mail: trnguyen@uottawa.ca,nthuongctu@yahoo.com,remi@uottawa.ca \\ Received March 14, 2011; revised April 8, 2011; accepted April 20, 2011
}

\begin{abstract}
We construct optimal k-step, 5- to 10-stage, explicit, strong-stability-preserving Hermite-Birkhoff (SSP HB) methods of order 12 with nonnegative coefficients by combining linear k-step methods of order 9 with 5- to 10-stage Runge-Kutta (RK) methods of order 4. Since these methods maintain the monotonicity property, they are well suited for solving hyperbolic PDEs by the method of lines after a spatial discretization. It is seen that the 8-step 7-stage HB methods have largest effective SSP coefficient among the HB methods of order 12 on hand. On Burgers' equations, some of the new HB methods have larger maximum effective CFL numbers than Huang's 7-step hybrid method of order 7, thus allowing larger step size.
\end{abstract}

Keywords: Strong Stability Preserving, Hermite-Birkhoff Method, SSP Coefficient, Time Discretization, Method of Lines, Comparison with Other SSP Methods

\section{Introduction}

We are concerned with the numerical solution of initial value problems

$$
\frac{\mathrm{d} y}{\mathrm{~d} t}=f(t, y(t)), \quad y\left(t_{0}\right)=y_{0} .
$$

where the function $f$ is such that

$$
\|y(t+\Delta t)\| \leq\|y(t)\|
$$

for all $\Delta t \geq 0$. Here $\|\cdot\|$ may be a norm or, more generally, any convex functional. It is also assumed that $f$ satisfies the discrete analog of (2),

$$
\left\|y_{n}+\Delta t f\left(t_{n}, y_{n}\right)\right\| \leq\left\|y_{n}\right\|, \quad \Delta t \leq \Delta t_{F E},
$$

for the forward Euler method. Here $y_{n}$ is a numerical approximation to $y\left(t_{0}+n \Delta t\right)$. We are interested in higher-order accurate multistep $\mathrm{HB}$ methods that preserve the monotonicity property

$$
\left\|y_{n}\right\| \leq \max _{1 \leq j \leq k}\left\|y_{n-j}\right\|,
$$

for $0 \leq \Delta t \leq \Delta t_{\max }=c \Delta t_{F E}$ whenever condition (3) holds. Here $k$ represents the number of previous steps used to compute the next solution value and $c$, called the SSP coefficient, depends only on the numerical integration method but not on $f$. The monotonicity property (4) is desirable as it mimics property (2) of the true solu- tion and prevents growth of errors.

Strong-stability-preserving (SSP) methods have been developed to satisfy the monotonicity property (4) for system (1) whenever condition (3) is fulfilled. The monotonicity property is guaranteed under the maximum time step $\Delta t_{\max }=c \Delta t_{F E}$. Considerable research effort has been devoted to find numerical methods with the largest value $c$ among various classes of methods.

The main application of such monotonicity results are found in the numerical solution of hyperbolic PDEs, in particular, of conservation laws. For the one-dimensional equation

$$
y_{t}+g(y)_{x}=0, \quad y(x, 0)=y_{0}(x),
$$

the spatial derivative $g(y)_{x}$ can be approximated by a conservative finite difference or finite element at $x_{j}, j$ $=1,2, \cdots, N$, (see, for example, [1-4]). This spatialsemidiscretization will lead to system (1) of ODEs.

In this paper, to solve system (1), we construct new explicit, multistep, multistage, SSP general linear timediscretization methods of order 12 with nonnegative coefficients. These methods, which we call SSP HermiteBirkhoff (SSP HB), because their construction involves HB interpolation polynomials (see Section 2), are combinations of linear $k$-step methods of order 9 and $s$-stage RK methods of order 4. The objective of high-order SSP HB time discretizations is to maintain the 
monotonicity property (4) while achieving higher-order accuracy in time, perhaps with a modified CFL restriction, measured here with an SSP coefficient, $c(H B k s)$ :

$$
\Delta t \leq c(H B k s) \Delta t_{F E},
$$

The SSP coefficient, historically called CFL coefficient, describes the ratio of the strong-stability- preserving time step to the strongly-stable forward Euler time step (see [5]). Since our arguments are based on convex decompositions of high- order methods in terms the SSP FE method, such high-order methods preserve SSP in any norm once FE is shown to be strongly stable.

Several new explicit 6- to 10-stage SSP HB methods with nonnegative coefficients presented here have been found by computer search.

On Burgers' equations, some of the new HB methods have larger maximum effective CFL numbers than Huang's 7-step hybrid method of order 7 [6], thus allowing larger step size.

In particular, no counterparts of $k$-step HB methods of order 12 have been found in the literature among hybrid and general linear multistep methods. Moreover, the 8-step, 7-stage HB method has largest effective SSP coefficient among the 12th-order HB methods on hand.

Section 2 introduces 5- to 10-stage SSP HB methods. Order conditions are listed in Section 3. Section 4 derives the Shu-Osher representation of $k$-step 5- to 10 -stage HB methods of order 12. New SSP HB methods are formulated as solutions of optimization problems in Section 5. Section 6 compares the effective SSP coefficients of different methods and lists several new SSP HB methods. Numerical results for several methods applied to Burgers' equations are presented in Section 7. Appendix A lists the Shu-Osher representation of some of the best HBks methods considered in this paper.

\section{2. $K$-step, $S$-stage SSP HB Methods of Order 12}

Notation 1: The following notation will be used:

- $k$ denotes the number of steps of a given method,

- $s$ denotes the number of stages of a given method,

- HBks denotes $k$-step, $s$-stage SSP HermiteBirkhoff methods of order 12,

- HMk denotes $k$-step SSP hybrid methods of order 7.

All HBks methods considered in this work are SSP and of order 12 unless specified otherwise. Therefore the denominations "SSP" and "order 12" will often be omitted in what follows.

Notation 2: The abscissa vector $\sigma=\left[c_{1}, c_{2}, c_{3}, \cdots\right.$, $\left.c_{s}\right]^{T}, \quad 0 \leq c_{j} \leq 1$, defines the off-step points $t_{n}+c_{j} \Delta t$.

An HBks method requires the following $s$ formulae to perform integration from $t_{n}$ to $t_{n+1}$, where, for simplicity, $c_{1}=0$ is used in the summations. By con- vention, $c_{1}^{0}=1$.

Let $F_{j}:=f\left(t_{n}+c_{j} \Delta t, Y_{j}\right)$ be the $j t h$ stage derivative and set $Y_{1}=y_{n}$.

An HB polynomial of degree $2 k+i-3$ is used as predictor $P_{i}$ to obtain the $i$ th stage value $Y_{i}$ to order 9,

$$
\begin{gathered}
Y_{i}=\sum_{j=0}^{k-1} \alpha_{i j} y_{n-j}+\Delta t\left[\sum_{j=1}^{i-1} a_{i j} F_{j}+\sum_{j=1}^{k-1} \beta_{i j} f_{n-j}\right], \\
i=2,3, \cdots, s .
\end{gathered}
$$

An HB polynomial of degree $2 k+s-2$ is used as integration formula to obtain $y_{n+1}$ to order 12:

$$
y_{n+1}=\sum_{j=0}^{k-1} \alpha_{j} y_{n-j}+\Delta t\left[\sum_{j=1}^{s} b_{j} F_{j}+\sum_{j=1}^{k-1} \beta_{j} f_{n-j}\right] \text {. }
$$

\section{Order Conditions of HBks}

To derive the order conditions for HBks we shall use the following expressions coming from the backsteps of the methods:

$$
\begin{gathered}
B_{i}(j)=\sum_{l=1}^{k-1} \alpha_{i l} \frac{(-l)^{j}}{j !}+\sum_{l=1}^{k-1} \beta_{i l} \frac{(-l)^{j-1}}{(j-1) !}, \\
j=1,2, \cdots, 12, \quad i=2,3, \cdots, s .
\end{gathered}
$$

As in the construction of RK methods, we impose the following simplifying conditions on the abscissa vector $\sigma=\left[c_{1}, c_{2}, c_{3}, \cdots, c_{s}\right]^{T}:$

$$
c_{i}=\sum_{j=1}^{i-1} a_{i l}+B_{i}(1), \quad i=2,3, \cdots, s .
$$

Forcing an expansion of the numerical solution produced by formulae (7)-(8) to agree with a Taylor expansion of the true solution, we obtain multistep- and RK-type order conditions that must be satisfied by $H B k s$. To reduce the large number of RK-type order conditions, we impose the following simplifying assumptions, as in similar searches for ODE solvers [7]:

$$
\begin{gathered}
\sum_{j=1}^{i-1} a_{i j} c_{j}^{k}+k ! B_{i}(k+1)=\frac{1}{k+1} c_{i}^{k+1}, \\
i=2,3, \cdots, s, \quad k=1,2, \cdots, 8 .
\end{gathered}
$$

Note that (11) with $k=0$ reduces to (10). There are seven sets of equations to be solved:

$$
\begin{gathered}
\sum_{j=0}^{k-1} \alpha_{i l}=1, \quad i=2,3, \cdots, s, \\
\sum_{i=0}^{k-1} \alpha_{i}=1, \\
\sum_{i=1}^{s} b_{i} c_{i}^{k}+k ! B_{i}(k+1)=\frac{1}{k+1}, \quad k=0,1, \cdots, 11,
\end{gathered}
$$




$$
\begin{gathered}
\sum_{i=2}^{s} b_{i}\left[\sum_{j=1}^{i-1} a_{i j} \frac{c_{i}^{9}}{9 !}+B_{i}(10)\right]+B(11)=\frac{1}{11 !}, \\
\sum_{i=2}^{s} b_{i} \frac{c_{i}}{11}\left[\sum_{j=1}^{i-1} a_{i j} \frac{c_{i}^{9}}{9 !}+B_{i}(10)\right]+B(12)=\frac{1}{12 !}, \\
\sum_{i=2}^{s} b_{i}\left[\sum_{j=1}^{i-1} a_{i j} \frac{c_{i}^{10}}{10 !}+B_{i}(11)\right]+B(12)=\frac{1}{12 !} \\
\sum_{i=2}^{s} b_{i}\left[\sum_{j=1}^{i-1} a_{i j}\left[\sum_{k=1}^{j-1} \alpha_{j k} \frac{c_{k}^{9}}{9 !}+B_{j}(10)\right]+B_{i}(11)\right] \\
+B(12)=\frac{1}{12 !}
\end{gathered}
$$

where the backstep parts, $B(j)$, are defined by

$$
\begin{gathered}
B(j)=\sum_{l=1}^{k-1} \alpha_{i} \frac{(-i)^{j}}{j !}+\sum_{l=1}^{k-1} \beta_{i} \frac{(-i)^{j-1}}{(j-1) !}, \\
j=1,2, \cdots, 12 .
\end{gathered}
$$

\section{Shu-Osher Representation of HBks}

We rewrite HBks in the Shu-Osher representation asconvex combinations of FE to show that they satisfy SSP conditions.

Firstly, if we let

$$
\begin{gathered}
\lambda_{i l} \geq 0, \sum_{j=1}^{i-1} \lambda_{i l}=1, i=3,4, \cdots, s, \\
\text { and } u_{s l} \geq 0, \sum_{l=1}^{s} u_{s l}=1,
\end{gathered}
$$

then formulae (7) and (8) become

$$
\begin{aligned}
& Y_{i}=\left[\sum_{l=1}^{i-1} \lambda_{i l}\right] \alpha_{i 0} y_{n}+\sum_{j=1}^{k-1} \alpha_{i j} y_{n-j} \\
&+\Delta t\left[\sum_{j=1}^{i-1} a_{i j} F_{j}+\sum_{j=1}^{k-1} \beta_{i j} f_{n-j}\right], i=3,4, \cdots, s, \\
& y_{n+1}=\left[\sum_{l=1}^{s} u_{s l}\right] \alpha_{o} y_{n}+\sum_{j=1}^{k-1} \alpha_{j} y_{n-j} \\
&+\Delta t\left[\sum_{j=1}^{i-1} b_{j} F_{j}+\sum_{j=1}^{k-1} \beta_{j} f_{n-j}\right]
\end{aligned}
$$

Replacing the index $i$ by $m$ in formula (7), we express $y_{n}$ as a function of $Y_{m}$,

$$
y_{n}=\frac{1}{\alpha_{m 0}}\left\{\begin{array}{c}
Y_{m}-\sum_{j=1}^{k-1} \alpha_{m j} y_{n-j}- \\
\Delta t\left[\sum_{j=1}^{m-1} a_{m j} F_{j}+\sum_{j=1}^{k-1} \beta_{m j} f_{n-j}\right] \\
m=2,3, \cdots, s,
\end{array}\right\},
$$

For $i=3,4, \cdots, s$ and $l=1,2, \cdots, i-1$, we replace the variable $y_{n}$ in the terms $\lambda_{i l} \alpha_{i 0} y_{n}$ in (21) by the right-hand side of (23) with $m=1$. Similarly, $y_{n}$ in the terms $u, \alpha_{0} y_{n}$ in (22) is replaced by the right-hand sides of (23) with $m=1$.

Secondly, we rewrite (7) with $i=2$, and (21) with $i=3,4, \cdots, s$ as (24), and (22) as (25) in the Shu-Osher equivalent form:

$$
\begin{aligned}
Y_{i}=\left[\sum_{j=0}^{k-1} A_{i j} y_{n-j}+\Delta t \sum_{j=0}^{k-1} B_{i j} f_{n-j}\right] \\
+\left[\sum_{j=2}^{i-1} e_{i j} Y_{j}+\Delta t \sum_{j=1}^{i-1} g_{i j} F_{j}\right], i=2,3, \cdots, s, \\
y_{n+1}=\left[\sum_{j=0}^{k-1} A_{j} y_{n-j}+\Delta t \sum_{j=0}^{k-1} B_{i} f_{n-j}\right] \\
+\left[\sum_{j=2}^{s} e_{j} Y_{j}+\Delta t \sum_{j=2}^{s} g_{j} F_{j}\right]
\end{aligned}
$$

where the coefficients are

$$
\begin{gathered}
A_{i j}=\alpha_{i, j}-\sum_{l=2}^{i-1} e_{i l} \alpha_{l j}, j=0,1, \ldots, k-1, i=2,3, \cdots, s, \\
A_{j}=\alpha_{j}-\sum_{l=2}^{s} e_{l} \alpha_{l j}, j=0,1 \cdots, k-1, \\
B_{i j}=\beta_{i, j}-\sum_{l=2}^{i-1} e_{i l} \beta_{l j}, j=0,1, \cdots, k-1, i=2,3, \cdots, s, \\
B_{j}=\beta_{j}-\sum_{l=2}^{s} e_{l} \beta_{l j}, j=0,1, \cdots, k-1, \\
g_{i j}=a_{i j}-\sum_{l=j+1}^{i-1} e_{i l} a_{l j}, i=3,4, \cdots, s, j=2,3, \cdots, i-1, \\
g_{j}=b_{j}-\sum_{l=j+1}^{i-1} e_{l} a_{l j}, j=2,3, \cdots, s,
\end{gathered}
$$

which follow from setting

$$
\begin{gathered}
e_{i j}=\lambda_{i j} \alpha_{i 0} / \alpha_{j 0}, j=2,3, \cdots, i-1, i=3,4, \cdots, s, \\
a_{i 1}=\beta_{i 0}, i=2,3, \cdots, s, \\
e_{j}=u_{s 1} \alpha_{0} / \alpha_{j 0}, j=2,3, \cdots, s, \\
b_{1}=\beta_{0} .
\end{gathered}
$$

Thirdly, the representation $(24,25)$, under the assumptions that all coefficients are nonnegative, implies that the $H B k p$ are SSP. In fact, one finds that the following functions are convex combinations of forward Euler steps:

- In (24) for $i=2,3, \cdots, s$, the first and second bracketed terms are sums of FE steps with step sizes $\frac{B_{i j}}{A_{i j}} \Delta t$, 
$j=0, \cdots, k-1$, and $\frac{g_{i j}}{e_{i j}} \Delta t, j=2,3, \cdots, i-1$, respectively.

- In (25), the first and second bracketed terms are sums of FE steps with step sizes $\frac{B_{i}}{A_{i}} \Delta t, j=0, \cdots, k-1$, and $\frac{g_{j}}{e_{j}} \Delta t, j=2,3, \cdots, i-1$, respectively.

One can easily verify that

$$
\sum_{j=0}^{k-1} A_{i j}+\sum_{j=2}^{i-1} e_{i j}=1, i=2,3, \cdots, s, \sum_{j=0}^{k-1} A_{j}+\sum_{j=2}^{s} e_{j}=1 .
$$

Provided all the coefficients $A_{i j}, e_{i j}, A_{j}, e_{j}$ are nonnegative, the following straightforward extension of a result presented in $[6,8]$ holds.

Theorem 1: If the forward Euler method FE is SSP under the CFL condition $\Delta t \leq \Delta t_{\mathrm{FE}}$, then the $k$-step, $s$-stage HBks methods $(24,25)$ are SSP provided

$$
\Delta t \leq c(H B k s) \Delta t_{F E},
$$

where the SSP coefficient $c(H B k s)$ is the minimum of the four numbers:

$$
\begin{array}{r}
\min _{j=0,1, \cdots, k-1}\left\{\frac{A_{j}}{B_{j}}\right\}, \min _{j=0,1, \cdots, k-1}\left\{\frac{A_{i j}}{B_{i j}}\right\}, i=2,3, \cdots, s, \\
\min _{\mathrm{j}=2,3, \cdots, s}\left\{\frac{e_{j}}{g_{j}}\right\}, \min _{j=2,3, \cdots, i-1}\left\{\frac{e_{i j}}{g_{i j}}\right\}, i=3,4, \cdots, s,
\end{array}
$$

with the convention that $\alpha / 0=+\infty$, under the assumption that all coefficients of (24) - (25) are nonnegative.

\section{Construction of Optimal HBks}

Since HBks contain many free parameters when $k$ is sufficiently large, we use the Matlab Optimization Toolbox to search for the methods with largest $c(H B k s)$ for different $k$ and $s$. To optimize HBks, we maximize $c$ (HBks) of Theorem 1 by solving the nonlinear programming problem

$$
\max _{A_{i j}, B_{i j}, e_{i j}, g_{i j}, A_{j}, B_{j}, e_{j}, g_{j}} c(H B k s),
$$

where all the numbers in all pairs

$$
\begin{gathered}
\left\{A_{j}, B_{j}\right\}, j=0,1, \cdots, k-1, \\
\left\{A_{i j}, B_{i j}\right\}, i=2,3, \cdots, s, j=0,1, \cdots, k-1, \\
\left\{e_{j}, g_{j}\right\}, j=2,3, \cdots, s, \\
\left\{e_{i j}, g_{i j}\right\}, i=3,4, \cdots, s, j=2,3, \cdots, i-1,
\end{gathered}
$$

are nonnegative. Null pairs, $\{0,0\}$, are not included in the minimization process if they occur. Besides the nonnegativity constraints on all variables, the objective func- tion (27) is subject to

- the convex combinations constraints (20),

- the simplifying assumptions (10) and (11) for HBks,

- the order conditions (12) to (18) for HBks,

- the conditions on the abscissa vector: $c_{1}=0$, $0 \leq c_{i} \leq 1, \quad i=2,3, \cdots, s$.

\section{Comparing Effective SSP Coefficients}

Definition 1: (See [9]) The effective SSP coefficients of an SSP method $\mathrm{M}$ is denoted by

$$
c_{\text {eff }}(M)=\frac{c(M)}{l}
$$

where $l$ is the number of function evaluations of method $M$ per time step and $c(M)$ is the SSP coefficient of $M$.

The SSP coefficients, $c(H M)$, of hybrid methods are defined in [6]. In this paper, $l=5,6, \cdots, 10$ for $H B$ methods and $l=2$ for hybrid methods. The numbers $c_{\text {eff }}(H B)$ and $c_{\text {eff }}(H M)$ will be used below.

The effective SSP coefficients, $c_{\text {eff }}$, provide a fair comparison between methods of the same order, although, in practice, starting methods and storage issues may also be important. Gottlieb [10] pointed out that one looks for highorder SSP methods $M$ with $c(M)$ as large as possible, taking their computational costs and orders into account.

We briefly review the developments of SSP methods. Shu and Osher [11] constructed a series of second- to fifth-order SSP RK methods, several of which are downwinded ones. Shu [12] found a class of first-order SSP RK methods with very large SSP coefficients, as well as one- to five-step SSP methods of orders two to five. Gottlieb and Shu [13] derived optimal $s$-stage SSP RK methods of order $s$ for $s=2,3$, and proved that for $s=4$ there is no such SSP method with nonnegative coefficients. Spiteri and Ruuth [14,15] studied optimal $s$-stage SSP RK methods of order $p$ with $s>p$ for $p \leq 4$. They proved the nonexistence of fifth order SSP RK methods with nonnegative coefficients [16] and constructed some fifth-order methods of seven to nine stages with downwind-biased spatial discretization [9]. A 10stage method of order 5 was given in [17]. Hunds-dorfer, Ruuth and Spiteri [18] proved that the implicit Euler method can unconditionally preserve the strong stability of the FE method (see also [19]) and studied multistep methods with specific starting procedures.

Ruuth and Hundsdorfer [20] pointed out that linear multistep methods of order five require at least seven steps. Huang [6] introduced the 7-step hybrid method HM 7 with $c(H M 7)=0.234$ and $c_{\text {eff }}(H M 7)=0.117$. 
Table 1. $c_{\text {eff }}(H B k s)$, for $k=7,8$, as a Function of $s$.

\begin{tabular}{ccc}
\hline$S$ & $C_{\text {eff }}(H B 7 s)$ & $c_{\text {eff }}(H B 8 s)$ \\
\hline 5 & 0.010 & 0.057 \\
6 & 0.035 & 0.091 \\
7 & 0.060 & 0.096 \\
8 & 0.055 & 0.091 \\
9 & 0.051 & 0.083 \\
10 & 0.047 & 0.076 \\
\hline
\end{tabular}

In the literature, we have found no general linear methods of order 12 . We now list our best methods.

HBk5 . Our best 5-stage SSP HBk5 method has step number $k=8$ with $c(H B 85)=0.288$ and $c_{\text {eff }}(H B 85)$ $=0.057$.

HBk6 . Our best 6-stage HBk6 has $k=8$ with $c(H B 86)=0.544$ and $c_{\text {eff }}(H B 86)=0.091$.

HBk7 . Our best 7 -stage HBk7 have $k=7,8$. Our HB87 has largest effective SSP coefficient among the 12th-order $H B$ methods on hand. The coefficients $c(H B 87)=0.669$ and $c_{\text {eff }}(H B 87)=0.096$ are listed in boldface in Table 1.

According to our search, it seems that $c_{\text {eff }}$ (HB87) cannot be improved up to 10 stages and 8 steps.

The formulae of some of our best new HBks are listed in Appendix A with their $c(H B k s), c_{e f f}(H B k s)$ and abscissa vector $\sigma$.

Table 1 lists $C_{\text {eff }}(H B k s)$ as a function of $s$ for $k=7,8$. We note that, for a given $k, c_{\text {eff }}(H B k s)$ first increases with $s$ and then decreases. We see that $c_{\text {eff }}(H B 87)=0.096$ is largest for the values of $k$ and $s$ on hand.

Definition 2: The percentage efficiency gain ( $P E G$ ) of the SSP coefficients $c_{\text {eff }}(M 2)$ of method 2 over $c_{\text {eff }}$ (M1) of method 1 is

$$
\operatorname{PEG}\left(c_{e f f}(M 2), c_{e f f}(M 1)\right)=\frac{c_{e f f}(M 2)-c_{e f f}(M 1)}{c_{e f f}(M 1)} .
$$

\section{Numerical Results}

From now on, we use the total variation semi-norm,

$$
T V\left(y_{n}\right)=\sum_{j}\left|y_{n, j+1}-y_{n, j}\right|,
$$

and say that a method is total variation diminishing (TVD) if

$$
T V\left(y_{n+1}\right) \leq T V\left(y_{n}\right) .
$$

We compare our new methods numerically with HM 7 of Huang.

\subsection{Starting Procedure}

To maintain the TVD property (31), the necessary starting values for HBkp were obtained by RK54 with small initial step size, $h_{1}=1.0 e-0.4$ (approximatively).

\subsection{Comparing HBks with HM7 with a Unit Downstep Initial Condition}

As a first comparison of HBks of order 12 with Huang's 7-step HM 7 of order 7 [6], we consider Burgers' equation in Problem 1.

Problem 1: Burgers' equation with a unit downstep initial condition:

$$
\begin{gathered}
\frac{\partial}{\partial t} u(x, t)+\frac{\partial}{\partial t}\left[\frac{1}{2} u(x, t)^{2}\right]=0, \\
u(x, 0)=\left\{\begin{array}{cc}
1, & -1 \leq x \leq 0, \\
0, & 0 \leq x \leq 1 .
\end{array}\right.
\end{gathered}
$$

and boundary condition $u(-1, t)=1$ for $t \geq 0$.

We discretize the spatial derivative of the flux function $f(u)=u(x, t)^{2} / 2$ by the weighted essentially nonoscillatory finite difference scheme of order 5 (WENO5) of Jiang and Shu [21] with spatial stepsize $\Delta x=1 / 150$. This leads to the semi-discrete system

$$
\frac{d}{d t} u_{j}(t)=-\frac{1}{\Delta x}\left[f_{j+(1 / 2)}-f_{j-(1 / 2)}\right],
$$

where $u_{j} \approx u\left(x_{j}, t\right)$ with $x_{j}=j \Delta x, j=-149, \cdots,-1$, $0,1, \cdots, 150$, and $f_{j+(1 / 2)}$ is the numerical flux, which typically is a Lipschitz continuous function of several neighboring values $u_{j}(t)$ (see [21] for details). A time discretization can then be applied to (33).

We consider the total variation norm of the numerical solution at $t_{\text {final }}=1.8$. For this purpose, we let $n_{\text {eff }}$ be the largest effective CFL number defined as

$$
n_{\text {eff }}=\max _{\Delta t}\left\{\frac{\Delta t}{\Delta x} \frac{1}{l}\right\},
$$

such that the TV error in the numerical solution satisfies the inequality

$$
\mid T V\left(u\left(x, t_{\text {final }}\right)\right)-T V(u(x, 0) \mid \leq 5.0 e-02,
$$

and we let $\max \Delta t_{\text {num }}=l \Delta x n_{\text {eff }}$ be the maximum numerical step size. Here $l$ is the number of function evaluations per time step. We note that Inequality (35) is used because $t_{\text {final }}$ is small.

Finally, we let max $\Delta t_{\text {theor }}$ of $H B k s$ for Problem 1 be taken as

$$
\max \Delta t_{\text {theor }}=c(H B k s) \Delta t_{\mathrm{FE}},
$$

where the SSP coefficients $c(H B k s)$ of some of the $H B k s$ are listed in Appendix A.

The numerical results for Problem 1 show that the forward Euler method, FE, has TVD property (31) with error (35) under the time step restriction 


$$
\Delta t \leq \Delta t_{\mathrm{FE}}=0.325 \Delta x .
$$

It was also observed numerically that the TVD property (31) holds with error (35) for the methods listed in Table 1 with $\Delta t \leq \max \Delta t_{\text {num }}$. This confirms the result of Theorem 1 that HBks are also TVD with $\Delta t \leq \max$ $\Delta t_{\text {num }}$ when combined with the WENO5 space discretization since HBks are convex combinations of $F E$. The same situation holds for Problem 2 below.

Definition 3: The $n_{\text {eff }}$ percentage efficiency gain of method 2 over method 1 is

$$
\operatorname{PEG}\left(n_{\text {eff }}\right)=\frac{n_{\text {eff }}(\operatorname{method} 2)-n_{\text {eff }}(\operatorname{method} 1)}{n_{\text {eff }}(\operatorname{method} 1)} .
$$

Table 2 lists PEG( $\left.n_{\text {eff }}(H B k s)\right), \quad n_{\text {eff }}(H M 7)$ for $H B k s$ and $n_{\text {eff }}(H M 7)=0.127$ for Problem 1. It is seen that

a) $n_{\text {eff }}(H B 8 s)>n_{\text {eff }}(H M 7)$ for all HB8s on hand,

b) quite remarkably, even though $c_{\text {eff }}(H B 8 s)<c_{\text {eff }}$ (HM 7), in this example, HB8s methods allow a larger step size since $n_{\text {eff }}(H B 8 s)>n_{\text {eff }}(H M 7)$,

c) $P E G\left(n_{\text {eff }}(H B 8 s)\right), \quad n_{\text {eff }}(H M 7)>0$ and increases as $S$ increases,

d) the ratios $R_{n / t}$ of $H B k s$ are clearly higher than the ratio of $H M 7$. For example, $R_{n / t}(H B 85)=7.310>$ $3.34=R_{n / t}(H M 7)$.

The $n_{\text {eff }}$ (HBks), for $k=7,8$, as a function of $s$ for Problem 1 are listed in Table 3.

\subsection{Comparing HBks and HM7 with a Square-Wave Initial Condition}

As a second comparison, we consider Burgers' equation with a square-wave initial value in Problem 2, which is test case 4 of Laney's five test problems [22, p.312].

Problem 2: Burgers' equation with a square wave initial condition:

$$
\begin{gathered}
\frac{\partial}{\partial t} u(x, t)+\frac{\partial}{\partial t}\left[\frac{1}{2} u(x, t)^{2}\right]=0, \\
u(x, 0)= \begin{cases}1, & |x| \leq \frac{1}{3} \\
0, & \frac{1}{3} \leq|x| \leq 1 .\end{cases}
\end{gathered}
$$

and boundary condition $u(-1, t)=0$ for $t \geq 0$.

We discretize the spatial derivative of Problem 2 by WENO5 and compute the total variation of the numerical solution as a function of the effective CFL number, $\Delta t /(l \Delta x)$, at $t_{\text {final }}=0.6$. In this case, $n_{\text {eff }}=0.325$ in the time step restriction (37) is replaced by $n_{\text {eff }}=0.183$.

Table 4 lists $P E G\left(n_{\text {eff }}(H B k s), n_{\text {eff }}(H M 7)\right)$ for HBks where $n_{\text {eff }}(H M 7)=0.122$ for Problem 2. It is seen that the results for Problem 2 listed in Table 4 con-
Table 2. PEG( $\left.n_{\text {eff }}(H B k s), n_{\text {eff }}(H M 7)\right)$ for HBks and $H M 7$, and ratio $R_{n / t}=\max \Delta t_{n u m} / \max \Delta t_{\text {theor }}$ for Problem 1. Here $n_{\text {eff }}(H M 7)=0.127$ and $R_{n / t}(H M 7)=3.340$.

\begin{tabular}{cccc}
\hline HBks & $n_{\text {eff }}(H B)$ & $R_{n / t}$ & $P E G$ \\
\hline$H B 85$ & 0.137 & 7.310 & $8 \%$ \\
$H B 86$ & 0.170 & 5.772 & $34 \%$ \\
$H B 77$ & 0.145 & 7.402 & $14 \%$ \\
$H B 87$ & 0.210 & 6.756 & $65 \%$ \\
\hline
\end{tabular}

Table 3. $n_{\text {eff }}($ HBks), for HBks HBks applied to Problem 1.

\begin{tabular}{ccc}
\hline$S$ & $n_{\text {eff }}(H B 7 s)$ & $n_{\text {eff }}(H B 8 s)$ \\
\hline 5 & 0.075 & 0.137 \\
6 & 0.084 & 0.170 \\
7 & 0.145 & 0.210 \\
\hline
\end{tabular}

Table 4. PEG( $\left.n_{\text {eff }}(H B k s), n_{\text {eff }}(H M 7)\right)$ for HBks and $H M 7$, and ratio $R_{\mathrm{n} / t}=\max \Delta t_{\text {num }} / \max \Delta t_{\text {theor }}$ for Problem 2. Here $n_{\text {eff }}(H M 7)=0.122$ and $R_{n / t}(H M 7)=5.689$.

\begin{tabular}{cccc}
\hline HBks & $n_{\text {eff }}(H B)$ & $R_{n / t}$ & $P E G$ \\
\hline HB85 & 0.137 & 12.999 & $12 \%$ \\
$H B 86$ & 0.158 & 9.541 & $30 \%$ \\
$H B 77$ & 0.138 & 12.536 & $14 \%$ \\
$H B 87$ & 0.203 & 11.608 & $67 \%$ \\
\hline
\end{tabular}

Table 5. $n_{e f f}(H B k s)$, for HBks applied to Problem 2.

\begin{tabular}{ccc}
\hline$s$ & $n_{\text {eff }}(H B 7 s)$ & $n_{\text {eff }}(H B 8 s)$ \\
\hline 5 & 0.075 & 0.137 \\
6 & 0.083 & 0.158 \\
7 & 0.138 & 0.20 \\
\hline
\end{tabular}

firm the observations (a-d) obtained for Problem 1 as listed in Table 2.

We observe that, as with hybrid methods, the ratio $\max \Delta t_{\text {num }} / \max \Delta t_{\text {theor }}$ of HBks for Problems 1 and 2 are greater than 1 . The theoretical strong-stability bounds of HBks are thus verified in the numerical comparison of the maximum time steps for Problem 1 and confirmed again with Problem 2.

Table 5 lists $n_{\text {eff }}$ (HBks) for $k=7,8$ as a function s for Problem 2.

\section{Conclusions}

New optimal explicit $k$-step, $s$-stage $(s=5,6, \ldots, 10)$ SSP Hermite-Birkhoff methods, HBks, of orders 12 with nonnegative coefficients are constructed by combining linear $k$-step methods of order 9 with 5- to 10-stage Runge-Kutta methods of order 4 . No counterparts of HBks of order 12 have been found in the literature among hybrid and general linear multistep methods. 
Moreover, the 8-step 7-stage HB87 has largest effective SSP coefficient among the 12th-order $H B$ methods on hand. It is found that some of new HBks have larger effective SSP coefficients and larger maximum effective CFL numbers than Huang's 7-step hybrid method of order 7 on Burgers' equations.

\section{References}

[1] A. Harten, "High Resolution Schemes for Hyperbolic Conservation Laws," Journal of Computational Physics Vol. 49, No. 3, 1983, pp. 357-393. doi:10.1016/0021-9991(83)90136-5

[2] S. Osher and S. Chakravarthy, "High Resolution Schemes and the Entropy Condition," SIAM Journal on Numerical Analysis, Vol. 21, No. 5, 1984, pp. 955-984. doi:10.1137/0721060

[3] P. K. Sweby, "High Resolution Schemes Using Flux Limiters for Hyperbolic Conservation Laws," SIAM Journal on Numerical Analysis, Vol. 21, No. 5, 1984, pp. 995-1011. doi:10.1137/0721062

[4] B. Cockburn and C. W. Shu, "TVB Runge-Kutta Local Projection Discontinuous Galerkin Finite Element Method for Conservation Laws II: General Framework," Mathematics of Computation, Vol. 52, No. 186, 1989, pp. 411-435.

[5] S. Gottlieb, D. I. Ketcheson and C. W. Shu, "High Order Strong Stability Preserving Time Discretization,” Journal of Scientific Computing, Vol. 38, No. 3, 2009, pp. 251-289.

[6] C. Huang, "Strong Stability Preserving Hybrid Methods," Applied Numerical Mathematics, Vol. 59, No. 5, 2009, pp. 891-904. doi:10.1016/j.apnum.2008.03.030

[7] T. Nguyen-Ba, E. Kengne and R. Vaillancourt, "One-Step 4-Stage Hermite-Birkhoff-Taylor ODE Solver of Order 12,” The Canadian Applied Mathematics Quarterly, Vol. 16, No. 1, 2008, pp. 77-94.

[8] S. Gottlieb, C. W. Shu and E. Tadmor, "Strong StabilityPreserving Highorder Time Discretization Methods," SIAM Review, Vol. 43, No. 1, 2001, pp. 8-112 doi:10.1137/S003614450036757X

[9] S. J. Ruuth and R. J. Spiteri, "High-Order Strong-Stability-Preserving Runge-Kutta Methods with Down-Biased Spatial Discretizations,” SIAM Journal on Numerical Analysis, Vol. 42, No. 3, 2004, pp. 974-996. doi:10.1137/S0036142902419284

[10] S. Gottlieb, "On High Order Strong Stability Preserving Runge-Kuttaand Multi Step Time Discretizations,” Journal of Scientific Computing, Vol. 25, No. 1-2, 2005, pp. 105-128.
[11] C. W. Shu and S. Osher, "Efficient Implementation of Essentially Nonoscillatory Shock-Capturing Schemes," Journal of Scientific Computing, Vol. 77, No. 2, 1988, pp. 439-471. doi:10.1016/0021-9991(88)90177-5

[12] C. W. Shu, "Total-Variation-Diminishing Time Discretizations,” SIAM Journal on Numerical Analysis, Vol. 9, No. 6, 1988, pp. 1073-1084. doi:10.1137/0909073

[13] S. Gottlieb and C. W. Shu, "Total Variation Diminishing Runge-Kutta Schemes," Mathematics of Computation, Vol. 67, No. 221, 1998, pp. 73-85. doi:10.1090/S0025-5718-98-00913-2

[14] R. J. Spiteri and S. J. Ruth, "A New Class of Optimal High-Order Strong-Stability-Preserving Time-Stepping Schemes,” SIAM Journal on Numerical Analysis, Vol. 40, No. 2, 2002, pp. 469-491. doi:10.1137/S0036142901389025

[15] R. J. Spiteri, S. J. Ruuh, "Nolinear Evoluton Using Optimal Fourth-Order Strong-Stability-Preserving RungeKutta Methods, Journal of Mathematics and Computers in Simulation, Vol. 62, No. 1-2, 2003, pp. 125-135. doi:10.1016/S0378-4754(02)00179-9

[16] S. J. Ruuth and R. J. Piteri, "Two Barriers on StrongStability-Preserving Time Discretization Methods," Journal on Scientific Computing, Vol. 17, No. 1-4, 2002, pp. 211-220. doi:10.1023/A:1015156832269

[17] S. J. Ruuth, "Global Optimization of Explicit StrongStability-Preserving Runge-Kutta Methods," Mathematics of Computation, Vol. 75, No. 253, 2006, pp. 183-207. doi:10.1090/S0025-5718-05-01772-2

[18] W. Hundsdorfer, S. J. Ruuth and R. J. Spiteri, "Monotonicity Preserving Linear Multistep Methods," SIAM Journal on Numerical Analysis, Vol. 41, No. , 2003, pp. 605-623. doi:10.1137/S0036142902406326

[19] I. Higueras, “On Strong Stability Preserving Methods," Journal of Scientific Computing, Vol. 21, No. , 2004, pp. 193-223. doi:10.1023/B:JOMP.0000030075.59237.61

[20] S. J. Ruuth and W. Hundsdorfer, "High-Order Linear Multistep Methods with General Monotonicity and Boundedness Properties," Journal of Computational Physics, Vol. 209, No. 1, 2005, pp. 226-248. doi:10.1016/j.jcp.2005.02.029

[21] G. Jiang and C. W. Shu, "Efficient Implementation of Weighted ENO Schemes," Journal of Computational Physics, Vol. 126, No. 1, 1996, pp. 202-228. doi:10.1006/jcph.1996.0130

[22] C. Laney, “Computational Gasdynamics,” Cambridge University Press, Cambridge, 1998. doi:10.1017/CBO9780511605604 


\section{Appendix}

This appendix lists the Shu-Osher representation of some of the best HBks methods considered in this paper with large $c(H B k s), C_{\text {eff }}(H B k s)$ and abscissa vector $\sigma=$ $\left[c_{1}, c_{2}, \ldots\right]$. For concision, $y_{-7}=y_{n-7}$ and $f_{-7}=f_{n-7}$, etc., that is, the $n$ is omitted.

HB85. $c=0.288, c_{\text {eff }}=0.0575$, and $\sigma=[0,0.26168792578970829,0.57645868591351512$, $0.64259830897152903,0.84768581516148367]^{T}$.

$$
\begin{aligned}
& Y_{2}=2.5088663215922309 e-3 y_{-7} \\
& +1.7925867066588250 e-1 y_{-6} \\
& +8.3882346204594546 e-2 y_{-5} \\
& +5.3490887614261351 e-2 y_{-4} \\
& +1.5355696110214626 e-1 y_{-3} \\
& +1.0519373621484439 e-1 y_{-2} \\
& +2.4588790492868035 e-1 y_{-1} \\
& +1.7622062694799823 e-1 y_{-0} \\
& +6.0965642854921308 e-2 h f_{-6} \\
& +2.91573176976848 e-1 h f_{-5} \\
& +1.8593314024575333 e-1 h f_{-4} \\
& +5.3376059470557180 e-1 h f_{-3} \\
& +3.6565109649432631 e-1 h f_{-2} \\
& +8.5470090983589497 e-1 h f_{-1} \\
& +6.1253899506765896 e-1 h f_{-0} \\
& Y_{3}=2.6128898286558854 e-4 y_{-7} \\
& +1.6160083267835362 e-1 y_{-6} \\
& +9.4862179832725935 e-2 y_{-5} \\
& +5.6365573282506388 e-3 y_{-4} \\
& +2.0977946829698155 e-1 y_{-3} \\
& +1.4916019106552419 e-2 y_{-2} \\
& +3.0292902279685230 e-1 y_{-1} \\
& +9.0823471553059566 e-4 h f_{-7} \\
& +3.7634075368106784 e-2 h f_{-6} \\
& +3.2973883540780097 e-1 h f_{-5} \\
& +1.9592548393933151 e-2 h f_{-4} \\
& +7.2918878409381671 e-1 h f_{-3} \\
& +5.1847751946960174 e-2 h f_{-2} \\
& +1.0529745717881731 e-0 h f_{-1} \\
& +2.1001463097741810 e-1 Y_{2} \\
& +7.3000620436093744 e-1 h F_{3} \text {, } \\
& Y_{4}=4.2596681035936669 e-3 y_{-7} \\
& +5.4580922815710267 e-2 y_{-6} \\
& +1.2619581254827076 e-2 y_{-5} \\
& +4.6309759376721930 e-2 y_{-4} \\
& +8.5864031653139994 e-2 y_{-2} \\
& +2.5760590697067362 e-2 y_{-1} \\
& +6.9756199296789845 e-1 y_{-0} \\
& +3.3666866480073193 e-2 h f_{-6} \\
& +4.3865384852407319 e-2 h f_{-5} \\
& +1.6097169759141286 e-1 h f_{-4} \\
& +2.9846147169135057 e-1 h f_{-2} \\
& +8.9543242531916173 e-2 h f_{-1}
\end{aligned}
$$

\footnotetext{
Copyright (C) 2011 SciRes.

$+5.2321449943060860 e-1 h f_{-0}$ $+7.3043453131041394 e-2 Y_{3}$ $+2.5389742479104255 e-1 h F_{3}$ $Y_{5}=1.9031575432429105 e-2 y_{-7}$ $+9.4390087983054396 e-3 y_{-6}$ $+4.9434657715702020 e-3 y_{-5}$ $+1.6566124692029068 e-2 y_{-4}$ $+1.1104031717607358 e-2 y_{-3}$ $+2.6842037578605454 e-2 y_{-2}$ $+1.8775366097652471 e-2 y_{-1}$ $+7.5605047579112805 e-1 y_{-0}$ $+5.4836011749628891 e-3 h f_{-7}$ $+3.2809785459763291 e-2 h f_{-6}$ $+1.7183377498495377 e-2 h f_{-5}$ $+5.7583482403654469 e-2 h f_{-4}$ $+3.8597368238337690 e-2 h f_{-3}$ $+9.3302327932468482 e-2 h f_{-2}$ $+6.5262756583411866 e-2 h f_{-1}$ $+3.5880557446886407 e-1 h f_{-0}$ $+1.3724791412067294 e-1 Y_{4}$ $+4.7707070872820062 e-1 h F$ $y_{n+1}=3.6675474446860911 e-4 y_{-7}$ $+1.8187060855967201 e-2 y_{-6}$ $+8.3994945677018339 e-3 y_{-5}$ $+4.9736412857637512 e-3 y_{-4}$ $+1.8712919442613503 e-2 y_{-3}$ $+1.6346596041633132 e-6 y_{-2}$ $+5.0971099921212137 e-1 y_{-1}$ $+1.6214093549108249 e-1 y_{-0}$ $+6.5806701062719308 e-3 h f_{-6}$ $+2.9196457024832888 e-2 h f_{-5}$ $+1.7288266917287534 e-2 h f_{-4}$ $+6.5045693394014711 e-2 h f_{-3}$ $+5.6820405678545405 e-6 h f_{-2}$ $+3.2159785087110521 e-1 h f_{-1}$ $+5.6359829950182783 e-1 h f_{-0}$ $+1.4048847659544447 e-1 Y_{2}$ $+1.1567333760542281 e-1 h F_{2}$ $+9.4775175249781230 e-11 Y_{3}$ $+4.2595421684290258 e-2 Y_{4}$ $+1.4806074206442218 e-1 h F_{4}$ $+9.4422661366167060 e-2 Y_{5}$ $+3.2821107895566276 e-1 h F_{5}$.

HB86. $c=0.544, c_{\text {eff }}=0.091$, $\sigma=[0,0.21131780320298513,0.44627527172505960$, 0.60194043865046165, 0.68474825481971757, $0.88846370798458207]^{T}$.

$Y_{2}=1.2056356845857071 e-4 y_{-7}$ $+2.5194380079479200 e-2 y_{-6}$ $+3.2952118982459200 e-2 y_{-5}$ $+1.1957054109007751 e-2 y_{-4}$ $+9.9102593302822214 e-2 y_{-3}$ $+6.2284629866456040 e-2 y_{-2}$ 


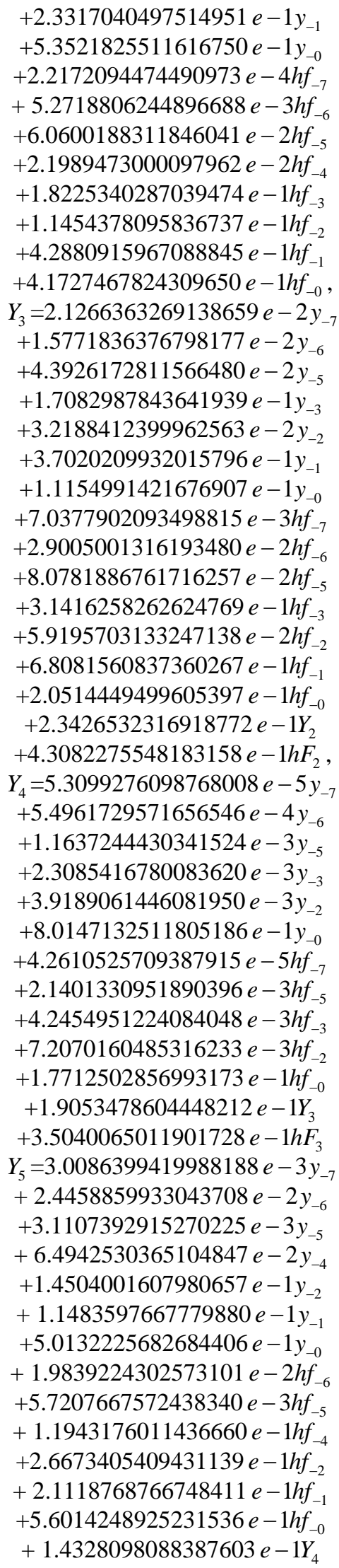

$$
\begin{aligned}
&+2.6349912209563475 e-1 h F_{4} \\
& Y_{6}=3.8364653662411327 e-4 y_{-7} \\
&+1.9538912222846316 e-2 y_{-6} \\
&+4.6039554350891257 e-2 y_{-5} \\
&+1.2188103769706057 e-1 y_{-3} \\
&+5.3347382574306705 e-2 y_{-2} \\
&+2.4574345904392714 e-1 y_{-1} \\
&+2.6965321938536446 e-1 y_{-0} \\
&+7.0554043510781647 e-4 h f_{-7} \\
&+2.6945695396165658 e-4 h f_{-6} \\
&+7.1279472038856595 e-2 h f_{-5} \\
&+2.2414382031142641 e-1 h f_{-3} \\
&+9.8107846468628329 e-2 h f_{-2} \\
&+4.5193147980540027 e-1 h f_{-1} \\
&+4.9590242989675859 e-1 h f_{-0} \\
&+2.4341278818897943 e-1 Y_{5} \\
&+4.4764528829286226 e-1 h F_{5} \\
& y_{n+1}=3.0057309944696390 e-4 y_{-7} \\
&+1.8418416885802473 e-2 y_{-6} \\
&+1.8025732157544459 e-2 y_{-5} \\
&+1.8990404477105753 e-2 y_{-4} \\
&+5.3350392235820249 e-2 y_{-3} \\
&+5.6588063024340191 e-2 y_{-2} \\
&+2.8208800017277152 e-1 y_{-1} \\
&+2.1570341210469796 e-1 y_{-0} \\
&+6.3394334033130204 e-3 h f_{-6} \\
&+3.3150000574699620 e-2 h f_{-5} \\
&+3.4924069315339902 e-2 h f_{-4} \\
&+9.8113381349543630 e-2 h f_{-3} \\
&+1.0406757991202498 e-1 h f_{-2} \\
&+3.3081996436653333 e-1 h f_{-1} \\
&+3.9668670169619830 e-1 h f_{-0} \\
&+9.1404198485699903 e-2 Y \\
&+1.6809576475720123 e-1 h F \\
&+1.2805484845172674 e-1 Y_{5} \\
&+2.3549769089357700 e-1 h F_{5} \\
&+1.1707595890504358 e-1 Y_{6} \\
&+2.1530709937689432 e-1 h F_{6} . \\
&+ 0.669 \\
& \hline
\end{aligned}
$$

HB87 . $c=0.669, c_{\text {eff }}=0.096$, and

$\sigma=[0,0.24630671392471543,0.34372959589592622$,

0.46965773869833194, 0.60188693905557489,

$0.74797892054340487,0.88945490483914114]^{T}$.

$$
\begin{aligned}
Y_{2} & =3.7974982255302016 e-3 y_{-7} \\
& +4.5089590734417921 e-2 y_{-5} \\
& +1.3429764576528219 e-1 y_{-3} \\
& +5.6617416306819886 e-2 y_{-2} \\
& +4.0351426372574184 e-1 y_{-1} \\
& +3.5668358524220800 e-1 y_{-0} \\
& +1.6821020062433984 e-3 h f_{-7} \\
& +4.1148530730146071 e-2 h f_{-5} \\
& +2.0060641782382357 e-1 h f_{-3} \\
& +8.4571974490169566 e-2 h f_{-2} \\
& +5.5717589231755449 e-1 h f_{-1}
\end{aligned}
$$


$+5.3279427144280755 e-1 h f_{-0}$, $Y_{3}=2.9172400973803583 e-5 y_{-7}$ $+1.5457113291505844 e-5 y_{-6}$ $+1.1177286333803951 e-4 y_{-5}$ $+2.8614723012174159 e-4 y_{-3}$ $+5.4885374083531169 e-4 y_{-2}$ $+8.5268194936899200 e-1 y_{-0}$ $+1.1450336076905814 e-5 f_{-7}$ $+2.3088983501061465 e-5 h f_{-6}$ $+1.6696013989213592 e-4 h f_{-5}$ $+4.2743095366881700 e-4 h f_{-3}$ $+8.1984745325029522 e-4 h f_{-2}$ $+9.0476868364274501 e-2 h f_{-0}$ $+1.4632664728244774 e-1 Y_{2}$ $+2.1857467698879354 e-1 h F_{2}$, $Y_{4}=3.8475518352648985 e-5 y_{-7}$ $+8.7276046172694814 e-5 y_{-6}$ $+3.9160153529198250 e-4 y_{-5}$ $+8.4698793426668008 e-4 y_{-3}$ $+1.9159825166593124 e-3 y_{-2}$ $+8.0838239885846730 e-1 y_{-0}$ $+2.0756050912562519 e-5 h f_{-7}$ $+5.8495277978687062 e-4 h f_{-5}$ $+1.2651838717277304 e-3 h f_{-3}$ $+2.8619890325691857 e-3 h f_{-2}$ $+1.2798384206338995 e-1 h f_{-0}$ $+1.8833727759078944 e-1 Y_{3}$ $+2.8132783999960803 e-1 h F_{3}$ $Y_{5}=3.945885121420493 e-4 y_{-7}$ $+3.0224741261588189 e-3 y_{-6}$ $+2.7859199581055545 e-5 y_{-5}$ $+1.1690557783204216 e-2 y_{-4}$ $+3.2441888015861495 e-2 y_{-2}$ $+2.6344135585813794 e-2 y_{-1}$ $+7.2046178550821049 e-1 y_{-0}$ $+2.5773136196436813 e-3 h f_{-6}$ $+4.1614536126434194 e-5 h f_{-5}$ $+3.9351417073771384 e-2 h f_{-1}$ $+2.4931199806475163 e-1 h f_{-0}$ $+2.0561671126902800 e-1 Y_{4}$ $+3.0713890520825726 e-1 h F_{4}$ $Y_{6}=1.1157847037721505 e-3 y_{-7}$ $+8.2716022628498732 e-3 y_{-6}$ $+3.1541447869261996 e-2 y_{-4}$ $+1.9317942446706354 e-3 y_{-3}$ $+8.3536788648073387 e-2 y_{-2}$ $+8.3768231333280554 e-2 y_{-1}$ $+5.5987874866806642 e-1 y_{-0}$ $+7.1766023733280809 e-3 h f_{-6}$ $+4.7114875573382890 e-2 h f_{-4}$ $+2.8856077199845985 e-3 h f_{-3}$ $+1.2478264850959904 e-1 h f_{-2}$ $+1.2512836483058420 e-1 h f_{-1}$
$+3.9923163881741003 e-1 h f_{-0}$ $+2.2995560227002496 e-1 Y_{5}$ $+3.4349499849412118 e-1 h F_{5}$ $Y_{7}=7.0665184156984342 e-5 y_{-7}$ $+1.1357098866877038 e-2 y_{-6}$ $+1.9489725260200538 e-2 y_{-5}$ $+1.1371636634757204 e-2 y_{-4}$ $+7.0530362148945702 e-2 y_{-3}$ $+6.7156684867183417 e-2 y_{-2}$ $+2.2796040165854331 e-1 y_{-1}$ $+2.5634355593341113 e-1 y_{-0}$ $+1.0555575548486729 e-4 h f_{-7}$ $+2.3467533365446628 e-3 h f_{-6}$ $+2.9112676894221846 e-2 h f_{-5}$ $+1.6986323751942745 e-2 h f_{-4}$ $+1.0535436580359381 e-1 h f_{-3}$ $+1.0031495271089685 e-1 h f_{-2}$ $+3.8291186859596027 e-1 h f_{-0}$ $+1.2182135533209280 e-1 Y_{3}$ $+1.8197002314043459 e-1 h F_{3}$ $+2.1389851411383179 e-1 Y_{6}$ $+3.1950980562391240 e-1 h F_{6}$ $y_{n+1}=1.8273596102868513 e-4 y_{-7}$ $+1.1489248786967506 e-2 y_{-6}$ $+1.4564465260880323 e-2 y_{-5}$ $+1.9394836900481112 e-2 y_{-4}$ $+5.2821409707040239 e-2 y_{-3}$ $+7.9212845840518423 e-2 y_{-2}$ $+1.9373980081939868 e-1 y_{-1}$ $+2.7692442403851641 e-1 y_{-0}$ $+3.9204470975736996 e-3 h f_{-6}$ $+2.1755595095175002 e-2 h f_{-5}$ $+2.8970937894792437 e-2 h f_{-4}$ $+7.8901709150237184 e-2 h f_{-3}$ $+1.1832378117387013 e-1 h f_{-2}$ $+2.8939783129338997 e-1 h f_{-1}$ $+4.1365443450426853 e-1 h f_{-0}$ $+5.2243622611154630 e-2 Y_{4}$ $+7.8038642646651585 e-2 h F_{4}$ $+1.3856684832443625 e-1 Y_{5}$ $+2.0698351719496980 e-1 h F_{5}$ $+1.6085976174957770 e-1 Y_{7}$ $+2.4028344199700516 e-1 h F_{7}$.

HB77 . $c=0.422, c_{\text {eff }}=0.060$, $\sigma=[0,0.24553329633115092,0.34381434970186381$, 0.46996349805312904, 0.59741148855197324, $0.74861321481234733,0.93726371152609467]^{T}$. $Y_{2}=7.3010787905497990 e-2 y_{-6}$ $+5.8928786071653340 e-2 y_{-5}$ $+3.2361722721541197 e-2 y_{-4}$ $+1.4144020818838035 e-1 y_{-3}$ $+9.4746286693606921 e-2 y_{-2}$ $+2.8032846082374741 e-1 y_{-1}$ 


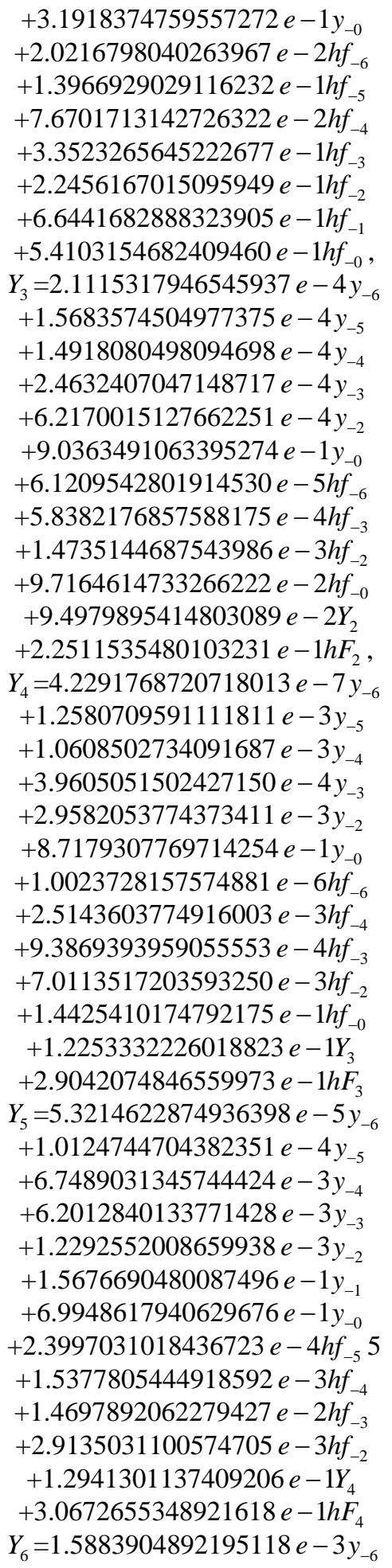

$+6.5792844833798811 e-2 y_{-5}$ $+4.3448235545907821 e-2 y_{-4}$ $+5.0430317113692086 e-2 y_{-3}$ $+9.0475494963689174 e-2 y_{-2}$ $+1.3063404640238935 e-1 y_{-1}$ $+4.6491915432714381 e-1 y_{-0}$ $+2.5214268095918969 e-2 h f_{-5}$ $+1.0297826627077401 e-1 h f_{-4}$ $+1.1952675542752805 e-1 h f_{-3}$ $+2.1443930908325182 e-1 h f_{-2}$ $+3.0962057366495105 e-1 h f_{-1}$ $+4.9881872517581938 e-1 h f_{-0}$ $+1.5271151632415950 e-1 Y_{5}$ $+3.6194719976664513 e-1 h F_{5}$ $Y_{7}=5.0565957696390162 e-3 y_{-6}$ $+4.2353916242658107 e-3 y_{-5}$ $+9.0603416352126546 e-3 y_{-4}$ $+1.8723784992724177 e-2 y_{-3}$ $+4.9888911059116470 e-1 y_{-1}$ $+2.7778379949012472 e-1 y_{-0}$ $+1.3708345905699068 e-3 h f_{-6}$ $+1.0038457971067822 e-2 h f_{-5}$ $+5.6604476160364535 e-3 h f_{-4}$ $+4.4377933703203629 e-2 h f_{-3}$ $+2.7937851702809663 e-1 h f_{-1}$ $+6.5838563316055287 e-1 h f_{-0}$ $+1.8625097589686893 e-1 Y_{6}$ $+4.4144031047782634 e-1 h F$ $y_{n+1}=1.0109380023947052 e-5 y_{-6}$ $+3.1517394957498736 e-3 y_{-5}$ $+2.7187605964756889 e-3 y_{-4}$ $+1.2707234915443108 e-1 y_{-2}$ $+7.5811479542621490 e-2 y_{-1}$ $+4.8361832605624699 e-1 y_{-0}$ $+8.7898513623300566 e-4 h f_{-5}$ $+6.4438348096906980 e-3 h f_{-4}$ $+6.2026529973672723 e-2 h f_{-2}$ $+1.7968358504392049 e-1 h f_{-1}$ $+3.1560924195242474 e-1 h f_{-0}$ $+1.4774510818353372 e-3 Y_{2}$ $+3.5017613257625627 e-3 h F_{2}$ $+1.6820128003980495 e-1 Y_{5}$ $+3.9866006030837425 e-1 h F$ $+6.5351056468600191 e-2 Y_{6}$ $+7.2587448184210451 e-2 Y_{7}$ $+1.7204218935729979 e-1 h F_{7}$. 